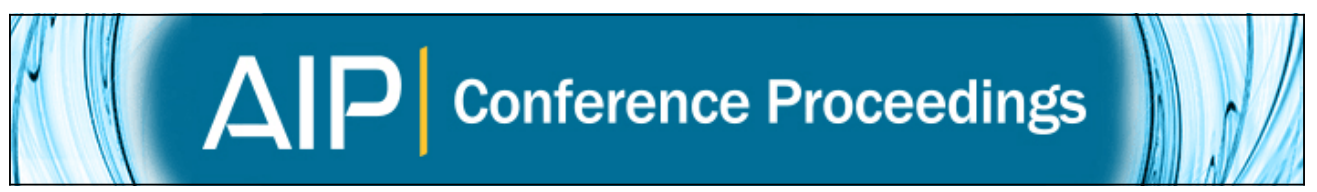

\title{
Jet correlations from unintegrated parton distributions
}

F. Hautmann and $\mathrm{H}$. Jung

Citation: AIP Conference Proceedings 1056, 79 (2008); doi: 10.1063/1.3013083

View online: http://dx.doi.org/10.1063/1.3013083

View Table of Contents:

http://scitation.aip.org/content/aip/proceeding/aipcp/1056?ver=pdfcov

Published by the AIP Publishing

Articles you may be interested in

The Angular Momentum Structure of the Nucleon

AIP Conf. Proc. 1056, 87 (2008); 10.1063/1.3013084

Study Of Short-Range Correlations With 6-9 GeV/c Protons

AIP Conf. Proc. 1056, 295 (2008); 10.1063/1.3013055

Studies of Parton Propagation and Hadron Formation in the Space-Time Domain AIP Conf. Proc. 1056, 215 (2008); 10.1063/1.3013044

Medium Modifications from $4 \mathrm{He}\left(\mathrm{e}^{\overrightarrow{1}}, \mathrm{e}^{\prime} \mathrm{p}^{\rightarrow}\right) 3 \mathrm{H}$

AIP Conf. Proc. 1056, 141 (2008); 10.1063/1.3013033

Generalized Parton Distributions, Analyticity and Formfactors

AIP Conf. Proc. 1056, 130 (2008); 10.1063/1.3013032 


\title{
Jet correlations from unintegrated parton distributions
}

\author{
F. Hautmann* and H. Jung ${ }^{\dagger}$ \\ * Oxford University, Theoretical Physics Department, Oxford OXI 3NP \\ ${ }^{\dagger}$ Deutsches Elektronen Synchrotron, D-22603 Hamburg
}

\begin{abstract}
Transverse-momentum dependent parton distributions can be introduced gaugeinvariantly in QCD from high-energy factorization. We discuss Monte Carlo applications of these distributions to parton showers and jet physics, with a view to the implications for the Monte Carlo description of complex hadronic final states with multiple hard scales at the LHC.
\end{abstract}

Keywords: Collider physics, jets, QCD.

PACS: 12.38.-t; 13.87.-a.

\author{
Sixth International Conference on Perspectives in Hadronic Physics \\ ICTP, Trieste, 12-16 May 2008 \\ AIP Conference Proceedings
}

\section{INTRODUCTION}

Experimental studies of multi-particle final states at high-energy hadron colliders rely on realistic event simulation by parton-shower Monte Carlo generators. Multi-particle states acquire qualitatively new features at the forthcoming Large Hadron Collider (LHC) compared to previous hadron-hadron experiments due to the large phase space opening up for events characterized by multiple hard scales $q_{1}^{2}, \ldots, q_{n}^{2}$, possibly widely disparate from each other. This brings in potentially large radiative corrections logarithmic in the ratio of two such scales, $\alpha_{s}^{k} \ln ^{m} q_{i}^{2} / q_{j}^{2}$, and potentially new effects in the nonperturbative components of production processes (e.g., parton densities being probed in regions of the phase space near the kinematic boundaries). It is not at all obvious that the approximations involved in standard Monte Carlo generators that have successfully served for event simulation in past collider experiments will be up to the new situation.

Standard parton-shower generators like HERWIG and PYTHIA are based on the dominance of collinear QCD radiation, supplemented by color-coherence effects for soft gluon emission from partons carrying longitudinal momentum fraction $x \sim \mathscr{O}(1)$. However as the energy increases the effects of emissions that are not collinearly ordered are known to become more and more important, and coherence effects from space-like partons carrying momentum fractions $x \ll 1$ set in. The high-energy multi-scale kinematics is bound to enhance the sensitivity to these dynamical features. The theoretical framework to take this into account requires the use of generalized QCD factorization 
techniques and the introduction of partonic distributions unintegrated not only in the longitudinal momenta but also in the transverse momenta. Although Monte Carlo implementations of this framework are not nearly as developed as standard shower generators at present, there are already studies that have been able to show their potential advantages over collinear-based algorithms in specific cases of hadronic final-state analyses.

In this article we briefly recall the basis for the introduction of transverse-momentum dependent (TMD) parton distributions from high-energy factorization, and point to ongoing activity toward fully general definitions; then we discuss Monte Carlo calculations that use the high-energy TMD framework to make predictions for jet observables, including angular and momentum correlations in final states with multiple jets. We comment on current developments toward general-purpose Monte Carlo tools and applications to final states with heavy quarks and heavy bosons plus jets at the LHC.

\section{TMD DISTRIBUTIONS FROM HIGH-ENERGY FACTORIZATION}

Precise definitions for transverse-momentum dependent (TMD), or unintegrated, parton distribution functions $[1,2]$ are at the center of much current activity. In the general case, to characterize such distributions gauge-invariantly over the whole phase space is a difficult question, and a number of open issues remain. In the case of small $x$ a well-prescribed, gauge-invariant definition emerges from high-energy factorization [3], and has been used for studies of collider processes both by Monte Carlo [4, 5] and by semi-analytic resummation [6, 7] approaches.

The diagrammatic argument for gauge invariance, given in [3], and developed in [8], is based on relating off-shell matrix elements with physical cross sections at $x \ll 1$, and exploits the dominance of single gluon polarization at high energies. ${ }^{1}$ The main reason why a natural definition for TMD pdfs can be constructed in the high-energy limit is that one can relate directly (up to perturbative corrections) the cross section for a physical process, say, photoproduction of a heavy-quark pair, to an unintegrated gluon distribution, much as, in the conventional parton picture, one does for DIS in terms of ordinary (integrated) parton distributions. On the other hand, the difficulties in defining a TMD distribution over the whole phase space can largely be associated with the fact that it is not obvious how to determine one such relation for general kinematics.

The evolution equations obeyed by TMD distributions defined from the high-energy limit are of the type of energy evolution [10]. Factorization formulas in terms of TMD distributions [3] have corrections that are down by logarithms of energy rather than powers of momentum transfer. On the other hand, it is important to observe that this framework allows one to describe the ultraviolet region of arbitrarily high $\mathrm{k}_{\perp}$ and in particular re-obtain the structure of QCD logarithmic scaling violations $[6,7,8]$. This ultimately justifies the use of this approach for jet physics. In particular it is the basis for using corresponding Monte Carlo implementations [11] to treat multi-scale hard processes at the LHC.

\footnotetext{
${ }^{1}$ It is emphasized e.g. in $[4,9]$ that a fully worked out operator argument, on the other hand, is highly desirable but is still missing.
} 
From both theoretical and phenomenological viewpoints, it is one of the appealing features of the high-energy framework for TMD distributions that one can relate its results to a well-defined summation of higher-order radiative corrections. By expanding these results to fixed order in $\alpha_{s}$, one can match the predictions thus obtained against perturbative calculations. This has been verified for a number of specific processes at next-to-leading order (see for instance [12] for heavy flavor production) and more recently at next-to-next-to-leading order (see for instance [13]). Note that this fact also provides the basis for shower algorithms implementing this framework to be combined with fixed-order NLO calculations by using existing techniques for such matching.

In the next section we point to topical issues and activity on TMD generalizations. After this, we focus on existing high-energy Monte Carlo with unintegrated pdfs and phenomenological applications to jets.

\section{LOW ENERGIES}

As mentioned above, in the general case full results on TMD distributions are yet to be established. The current status is discussed in [1]. Factorization formulas in terms of unintegrated parton distributions will have a considerably complex structure. A prototypical calculation that illustrates this structure is carried out in [14], which treats, rather than a general scattering observable, a simpler problem, the electromagnetic form factor of a quark. This case is however sufficient to illustrate certain main features, namely the role of nonperturbative, gauge-invariantly defined factors associated with infrared subgraphs (both collinear and soft), and the role of infrared subtractive techniques that serve to identify these factors. Analyses along these lines for more general processes, involving fully unintegrated pdfs, have recently been reported by T. Rogers [15].

One of the questions that a full factorization statement will address is the treatment of soft gluons exchanged between subgraphs in different collinear directions. The underlying dynamics is that of non-abelian Coulomb phase, treated a long time ago in [16] for the fully inclusive Drell-Yan case. But a systematic treatment for more complex observables, including color in both initial and final states, is still missing. Vogelsang and Yuan [17] illustrate this point neatly with a simplified calculation for di-jet hadroproduction near the back-to-back region. ${ }^{2}$

A further question concerns lightcone divergences and the $x \rightarrow 1$ endpoint behavior. The singularity structure at $x \rightarrow 1$ is different in the TMD case than for ordinary (integrated) distributions, giving divergences even in dimensional regularization with an infrared cut-off [19]. The singularities can be understood in terms of gauge-invariant eikonal-line matrix elements [19], and the TMD behavior can be related to cusp anomalous dimensions $[20,21]$ and lack of complete $\mathrm{KLN}$ cancellations [2, 20, 22]. In general this affects the precise form of factorization and relation with collinear distributions.

\footnotetext{
2 Note that interestingly in [18], which has a different point of view than TMD, Coulomb/radiative mixing terms are found to be responsible for the breaking of angular ordering in the initial-state cascade and the appearance of superleading logarithms in di-jet cross sections with a gap in rapidity.
} 
Relevant applications are both at low energies and at high energies. An important example for current experimental programs is semi-inclusive leptoproduction ([23, 24, 25], and references therein), where infrared subtractive techniques of the type $[14,26]$ serve for TMD-factorization calculations [27], and in particular for the proper treatment of overlapping momentum regions. ${ }^{3}$ Implications for spin asymmetries [34] and possibly exclusive reactions [35] are being studied. General characterizations of TMD distributions will be relevant at colliders for turning present $\mathrm{k}_{\perp}$-showering generators into general-purpose tools to describe hadronic final states over the whole phase space $[5,36]$.

In the next section we consider applications of $\mathrm{k}_{\perp}$-shower generators to multi-jet final states [37]. The main focus is on regions where jets are far from back-to-back, and the total energy is much larger than the transferred momenta so that the values of $x$ are small. In this regime the ambiguities related to soft Coulomb exchange and to lightcone divergences are not expected to be crucial. We find that the TMD distributions, as well as the transverse-momentum dependence of short-distance matrix elements, play a very essential role to describe correlations in angle and momentum of the jets.

\section{MULTI-JET CORRELATIONS}

This section presents multi-jet results [37] of Monte Carlo implementing TMD distributions according to high-energy factorization, and compares them with collinear-based Monte Carlo results.
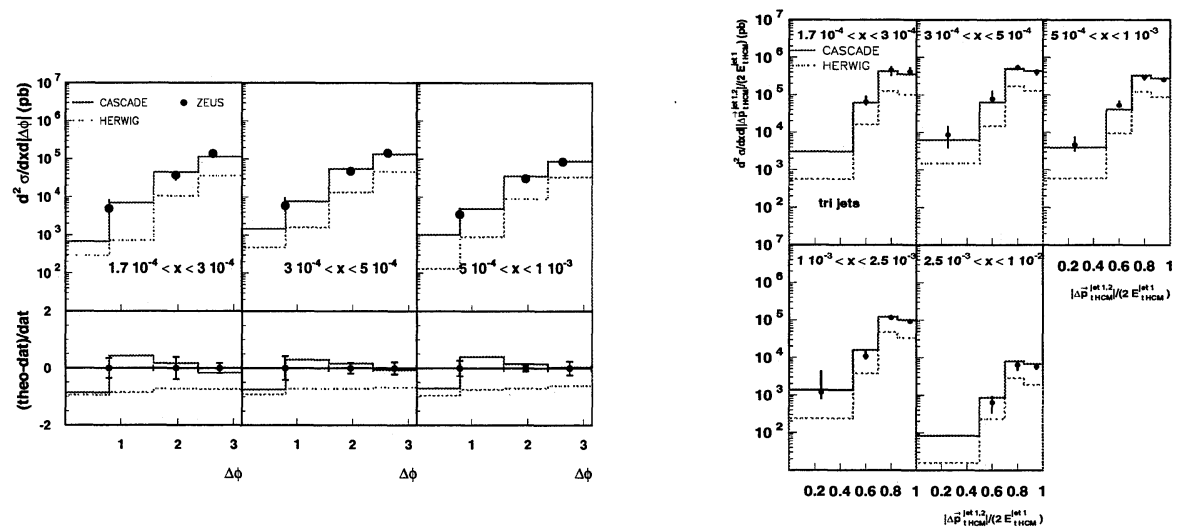

FIGURE 1. (left) Angular correlations and (right) momentum correlations [37] in three-jet final states measured by [38], compared with the CASCADE and HERWIG Monte Carlo results.

In Fig. 1 we consider three-jet production in $e p$, for which precise and interesting measurements have recently appeared [38], and we show results for the cross section

\footnotetext{
${ }^{3}$ Subtraction techniques related to those of $[14,26]$ are developed in [28] for soft-collinear effective theory, and studied in [29] and [30] in relation with standard perturbative methods. See also SCET applications to shower algorithms [31], TMD pdfs [32] and jet event shapes [33] for use of these techniques.
} 
in the azimuthal separation $\Delta \phi$ between the two leading jets and in the transversemomentum imbalance $\Delta p_{t}$. The shape of the distributions is different for HERWIG and for the $\mathrm{k}_{\perp}$-shower Monte Carlo CASCADE [39], with the largest differences occurring at small $\Delta \phi$ and small $\Delta p_{t}$, where the jets are not close to back-to-back configurations [40] and one has three hard, well-separated jets. ${ }^{4}$ By analyzing the angular distribution of the third jet, Refs. [5, 37] find significant contributions from regions where the transverse momenta in the initial state shower are not ordered. The description of the measurement by the $\mathrm{k}_{\perp}$-shower is good, whereas the collinear-based shower is not sufficient to describe it.

Note that the interpretation $[5,37]$ of the angular correlation data in terms of corrections to collinear ordering is consistent with the finding in [38] that while inclusive jet rates are reliably predicted by NLO fixed-order results, NLO predictions are affected by large corrections to di-jet azimuthal distributions (going from $\mathscr{O}\left(\alpha_{s}^{2}\right)$ to $\mathscr{O}\left(\alpha_{s}^{3}\right)$ ) in the small- $\Delta \phi$ and small- $x$ region, and begin to fall below the data for three-jet distributions in the smallest $\Delta \phi$ bins.

It is important to realize that the result in Fig. 1 receives contribution from the transverse-momentum dependence of both TMD pdfs and hard matrix elements. Fig. 2 shows different approximations to the azimuthal dijet distribution normalized to the back-to-back cross section. The solid red curve is the full result. The dashed blue curve is obtained from the same TMD pdfs but not including the transverse-momentum dependence of the hard ME. We see that the high- $\mathrm{k}_{\perp}$ component in the hard ME [3] is essential to describe jet correlations for small $\Delta \phi$. For reference we also plot with the dotted (violet) curve the result obtained from the TMD pdf without any resolved branching, corresponding to nonperturbative, predominantly low- $\mathrm{k}_{\perp}$ modes.

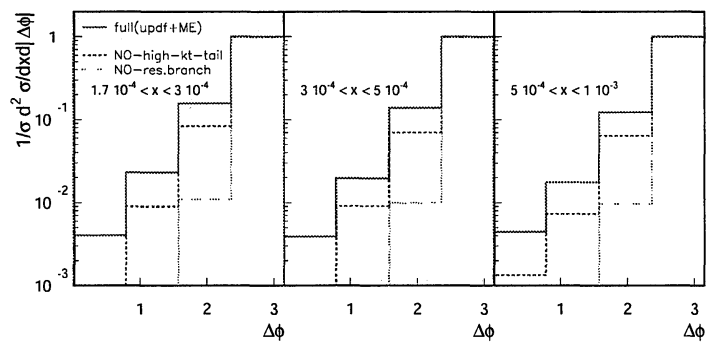

FIGURE 2. Azimuthal distribution normalized to the back-to-back cross section [37]: (solid red) full result; (dashed blue) same TMD pdfs but no finite- $\mathrm{k}_{\perp}$ correction in ME; (dotted violet) TMD pdfs with no resolved branching.

To examine more closely the distribution in $\mathrm{k}_{\perp}$ that results from highly off-shell subprocesses, in Fig. 3 we study the jet cross section in transverse energy and compare the $\mathrm{k}_{\perp}$-shower with the NLO result. It is noteworthy that the large- $p_{t}$ part of the di-jet spectrum is very close for the two calculations. At low $p_{t}$ one sees the Sudakov formfactor effect in the shower result. Differences in the single-jet spectra are also of interest

\footnotetext{
${ }^{4}$ Near $\Delta \phi \sim \pi$, on the other hand, soft-gluon exchange effects such as in [17] may well affect the predictions.
} 
and currently under study. This may be of use to relate [41] DIS event shapes measuring the transverse momentum in the current region to hadro-production $p_{T}$ spectra.
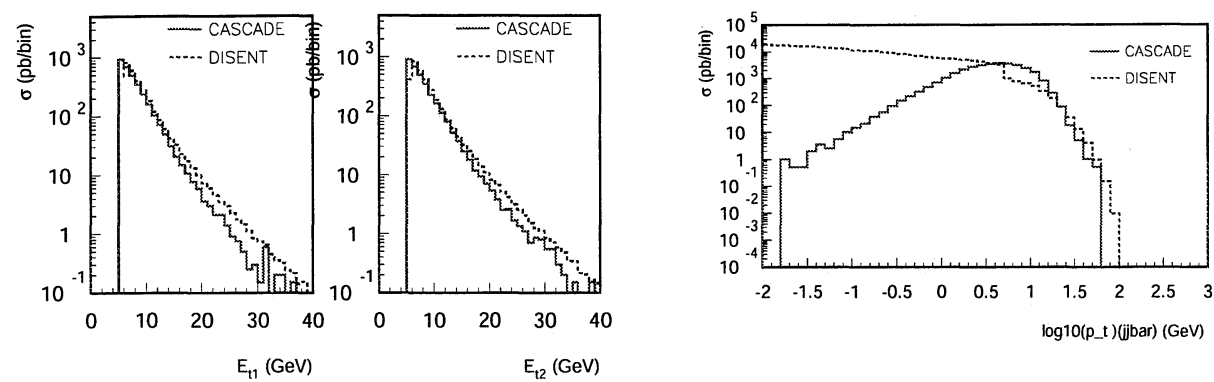

FIGURE 3. Comparison of the $\mathrm{k}_{\perp}$-shower CASCADE with the NLO di-jet calculation DISENT: (left) distribution in single-jet transverse energy; (right) distribution in the di-jet transverse energy.

Let us note that besides jet final states the off-shell effects mentioned above are expected also for heavy mass production. For instance, they may affect the phenomenological small- $x$ broadening of $\mathrm{W}$ and $\mathrm{Z} p_{T}$ distributions emphasized by Olness [42] (see [43]), and their use as luminosity monitor [44]. Multi-scale effects may arise [45] in the associated production of $\mathrm{W}$ and bottom quark pairs [46] and in final states with Higgs $[47,48]^{5}$ especially for measurements of non-inclusive observables and correlations.

\section{SUMMARY AND PROSPECTS FOR LHC FINAL STATES}

We have discussed the method of $\mathrm{k}_{\perp}$-dependent Monte Carlo shower, based on transverse-momentum dependent (TMD), or unintegrated, parton distributions and matrix elements defined by high-energy factorization. The theoretical basis of the method allows one to go to arbitrarily high transferred-momentum scales, thus making it suitable for the simulation of hard processes at the LHC.

We have pointed to developments of the approach toward general-purpose event generators, and illustrated applications to experimental $e p$ data for final states with multiple hadronic jets. Despite the lower ep energy, the multi-jet kinematic region considered is characterized by large phase space available for jet production and is relevant for extrapolating to the LHC initial-state showering effects.

The multi-scale QCD effects that we are treating also affect heavy mass production at the LHC, including final states with heavy bosons and heavy flavor. It is interesting to note that even at LHC energies the transverse momentum distribution of top quark pairs calculated from the $\mathrm{k}_{\perp}$-shower is similar to what is obtained from a full NLO calculation (including parton showers. MC@NLO [50]), where the $\mathrm{k}_{\perp}$-shower predicts an even harder spectrum, Fig. 4.

${ }^{5}$ Similar effects were noted [49] in the predictions for the Higgs transverse-momentum spectrum at the LHC. 
We conclude by observing that, using unintegrated parton distributions together with the off-shell matrix elements, many of the sub-leading effects are properly simulated, both in $e p$ collisions at HERA as well as at the LHC. For ep we could show that the predictions coming from the $\mathrm{k}_{\perp}$-shower simulation CASCADE are in good agreement with the measurements. For the LHC even at the large scale of $t \bar{t}$ production we observe reasonable comparison with results containing full NLO effects.

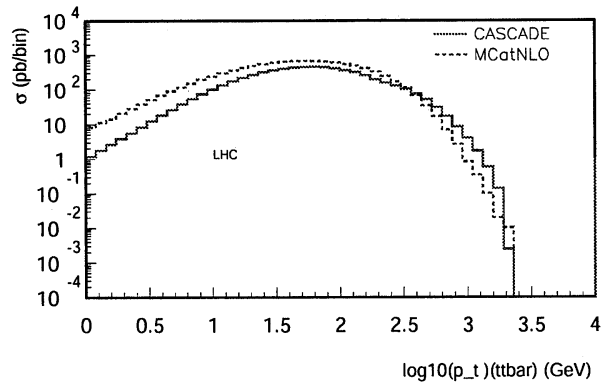

FIGURE 4. Comparison of transverse momentum distribution of $t \bar{t}$ pairs calculated from the $\mathrm{k}_{\perp}$-shower CASCADE with the NLO calculation MC@NLO at LHC energies.

\section{ACKNOWLEDGMENTS}

It is a pleasure to thank the organizers of the conference for the invitation and the opportunity to participate in a very interesting and enjoyable meeting. We wish to thank J. Collins, M. Mangano and J.W. Qiu for useful discussions.

\section{REFERENCES}

1. J.C. Collins, talk at the Light Cone 2008 Workshop, Mulhouse, July 2008.

2. J.C. Collins, Acta Phys. Polon. B 34 (2003) 3103.

3. S. Catani, M. Ciafaloni and F. Hautmann, Nucl. Phys. B366 (1991) 135; Phys. Lett. B 242 (1990) 97.

4. J.R. Andersen et al., Eur. Phys. J. C 35 (2004) 67.

5. F. Hautmann and H. Jung, arXiv:0712.0568 [hep-ph].

6. M. Ciafaloni, talk at RADCOR2007, Florence, October 2007.

7. G. Altarelli, talk at RADCOR2007, Florence, October 2007 [arXiv:0802.0968 [hep-ph]].

8. S. Catani and F. Hautmann, Nucl. Phys. B427 (1994) 475; S. Catani, M. Ciafaloni and F. Hautmann, Phys. Lett. B 307 (1993) 147.

9. J.C. Collins, hep-ph/0106126, in Proceedings of the Workshop DIS01 (Bologna, 2001).

10. L.N. Lipatov, Phys. Rept. 286 (1997) 131.

11. H. Jung, Mod. Phys. Lett. A19 (2004) 1.

12. M. Buza, Y. Matiounine, J. Smith, R. Migneron and W.L. van Neerven, Nucl. Phys. B472 (1996) 611; S. Riemersma, W.L. van Neerven and and J. Smith, Phys. Lett. B 347 (1995) 143; E. Laenen, S. Riemersma, W.L. van Neerven and and J. Smith, Nucl. Phys. B392 (1993) 162.

13. S. Moch, J.A.M. Vermaseren and A. Vogt, Phys. Lett. B 606 (2005) 123; Nucl. Phys. B691 (2004) 129.

14. J.C. Collins and F. Hautmann, Phys. Lett. B 472 (2000) 129.

15. T.C. Rogers, arXiv:0807.2430 [hep-ph]; arXiv:0712.1195 [hep-ph], in Proceedings of the 8th International Symposium on Radiative Corrections RADCOR2007 (Florence, October 2007). 
16. J.C. Collins, D.E. Soper and G. Sterman, Nucl. Phys. B308 (1988) 833.

17. W. Vogelsang and F. Yuan, Phys. Rev. D 76 (2007) 094013; and references therein.

18. M.H. Seymour, arXiv:0710.2733 [hep-ph]; J.R. Forshaw, A. Kyrieleis and M.H. Seymour, JHEP 0608 (2006) 059.

19. F. Hautmann, Phys. Lett. B 655 (2007) 26.

20. G.P. Korchemsky and A. Radyushkin, Phys. Lett. B 279 (1992) 359, G.P. Korchemsky and G. Marchesini, Phys. Lett. B 313 (1993) 433.

21. I.O. Cherednikov and N.G. Stefanis, Nucl. Phys. B802 (2008) 146; Phys. Rev. D 77 (2008) 094001; arXiv:0711.1278 [hep-ph].

22. J.C. Collins, in Perturbative Quantum Chromodynamics, ed. A.H. Mueller, World Scientific 1989, p. 573.

23. M. Anselmino et al., arXiv:0807.0173 [hep-ph]; M. Anselmino et al., Phys. Rev. D 71 (2005) 074006.

24. F. Ceccopieri and L. Trentadue, Phys. Lett. B 660 (2008) 43.

25. A. Bacchetta, D. Boer, M. Diehl and P.J. Mulders, arXiv:0803.0227 [hep-ph].

26. J.C. Collins and F. Hautmann, JHEP 0103 (2001) 016; F. Hautmann, hep-ph/0101006.

27. X. Ji, J. Ma and F. Yuan, Phys. Rev. D 71 (2005) 034005, JHEP 0507 (2005) 020.

28. A.V. Manohar and I.W. Stewart, Phys. Rev. D 76 (2007) 074002.

29. C. Lee and G. Sterman, Phys. Rev. D 75 (2007) 014022.

30. A. Idilbi and T. Mehen, Phys. Rev. D 75 (2007) 114017.

31. C.W. Bauer, F.J. Tackmann and J. Thaler, arXiv:0801.4028; arXiv:0801.4026.

32. J. Chay, arXiv:0711.4295.

33. C.W. Bauer, S.P. Fleming, C. Lee and G. Sterman, arXiv:0801.4569 [hep-ph]; J. Chiu, F. Golf, R. Kelley and A.V. Manohar, Phys. Rev. D 77 (2008) 053004; M.D. Schwartz, Phys. Rev. D 77 (2008) 014026; M. Trott, Phys. Rev. D 75 (2007) 054011.

34. Y. Koike, W. Vogelsang and F. Yuan, Phys. Lett. B 659 (2008) 878; and references therein.

35. S. Meissner, K. Goeke, A. Metz and M. Schlegel, arXiv:0805.3165 [hep-ph]; and references therein.

36. F. Hautmann, arXiv:0708.1319 [hep-ph].

37. F. Hautmann and H. Jung, arXiv:0805.1049 [hep-ph]; arXiv:0804.1746 [hep-ph], in Proceedings of the 8th International Symposium on Radiative Corrections RADCOR2007 (Florence, October 2007).

38. S. Chekanov et al. [ZEUS Collaboration], Nucl. Phys. B 786 (2007) 152 [arXiv:0705.1931 [hep-ex]].

39. H. Jung, Comput. Phys. Commun. 143 (2002) 100.

40. Y. Delenda, arXiv:0706.2172; A. Banfi, M. Dasgupta and Y. Delenda, Phys. Lett. B 665 (2008) 86.

41. M. Dasgupta and Y. Delenda, JHEP 0608 (2006) 080.

42. F.I. Olness, talk at HERA-LHC Workshop, CERN, May 2008.

43. S. Berge, P.M. Nadolsky, F.I. Olness and C.P. Yuan, hep-ph/0508215; P.M. Nadolsky, N. Kidonakis, F.I. Olness and C.P. Yuan, Phys. Rev. D 67 (2003) 074015.

44. A.M. Cooper-Sarkar, arXiv:0707.1593 [hep-ph].

45. M. Deak and F. Schwennsen, arXiv:0805.3763 [hep-ph].

46. M.L. Mangano, Nucl. Phys. B 405 (1993) 536.

47. F. Hautmann, Phys. Lett. B $\mathbf{5 3 5}(2002) 159$.

48. H. Jung, Mod. Phys. Lett. A 19 (2004) 1 [arXiv:hep-ph/0311249].

49. A. Kulesza, G. Sterman and W. Vogelsang, Phys. Rev. D 69 (2004) 014012.

50. S. Frixione, P. Nason and B. R. Webber, JHEP 0308 (2003) 007 [arXiv:hep-ph/0305252]. 